\title{
The reward and risk of social media for academics
}

Ben Britton ${ }^{1}$, Chris Jackson², Jessica Wade ${ }^{3}$

1. Department of Materials, Imperial College London

2. Department of Earth Sciences \& Engineering, Imperial College London

3. Department of Physics, Imperial College London

\section{Standfirst (50 words max):}

We are three academics who are active on social media. We explore the motivations for and benefits of engaging with social media, as well as its costs and risks. Overall, we believe this engagement to be a net benefit for us, our employers and for wider society.

\section{Main Article}

For better or for worse, society has been transformed by social media. But academics who engage in this way must face some obvious dilemmas: How much time should be spent on social media? Does a social media presence erode academic authority? Is it possible to evaluate the impact of our online activities?

For the three of us, the social media we engage with most is Twitter. For the unfamiliar, Twitter is a popular microblogging service that enables sharing of short ( $<280$ characters), largely text-based messages (videos, links and images are also popular). We should come clean: although we all work in the same institution, Twitter is where we 'met'.

Our interactions on social media have enriched both our academic and personal lives. You may reasonably wonder if Twitter is a good use of our time. One of the joys of being academics is that, within reason, our time is our own. Provided we deliver on the core requirements determined by our institution, what we do in addition to that is up to us. Spending time on social media is little different to spending time chatting to colleagues or friends in a café. But social media provides a 24/7 café that transcends lab walls, country borders, career stages, languages (through Twitter's in-built translation tools), cultures, and backgrounds. The benefits of social media are timely and immediate: it helps us to communicate with our peers without the need (or environmental cost) of flying to international conferences. It can help reduce the feeling of isolation. It creates inclusive spaces that facilitate interactions with a diverse range of people, particularly those from a generation that have grown up online. Our interactions have also helped us recruit new staff and students.

In this article, we argue that our engagements on social media benefit our scientific and teaching activities and, in turn, benefit our employers. Social media platforms provide us with the space to analyse and develop our academic practise. We can share teaching styles, discuss academic culture, and build interdisciplinary collaborations that could not happen without an accessible, informal, non-specialist, international and truly open forum. Extended benefits include meaningful interactions outside of academia that improve the public engagement profile of our institution.

On social media, our frequent interactions are not only with fellow academics, but with journal editors, policy makers, journalists, school teachers, alumni, students, pupils, parents, people in business and individuals from industry. Who interacts with who on Twitter is 
complicated ${ }^{1,2}$, however research indicates that the academic social media 'bubble' can be popped easily once a user has a sufficiently large following ${ }^{3}$. And while these interactions may be virtual on Twitter, they occasionally form the basis of our next papers, talks or grant proposals, and support committee work. Furthermore, an entire poster conference is now run by the Royal Society of Chemistry on Twitter ${ }^{4}$.

Beyond these shared examples, each of us can cite personal benefits of our engagement on social media. Chris engages with classrooms of African-American high school students who, having seen his recent TV documentaries, are inspired to continue in their exploration of the world through science. By sharing these experiences on Twitter, Chris highlights the value of senior academics engaging with the public and the importance of diverse role models. Jess shares her passion to raise the profile of these role models, though her creation of over 600 Wikipedia biographies for scientists from underrepresented groups, while enthusiastically training and supporting new Wikipedia editors. Ben openly shares the challenges of being LGBTQ+ in STEM, as well as the importance and reward of listening to and learning from members of marginalized groups.

Alongside our daily observations, Twitter enables us to campaign for better access and equity in science. For example, recent social media activity has led (with many others) to the formation of The Inclusion Group for Equity in Research in STEMM (TIGERS, @TIGERSinSTEMM). The TIGERS connect on Twitter, share information on Slack (an eplatform for discussions, connections and commerce), use a JISC mailling list, as well as use a conventional website. As a group, the TIGERS have recently launched an inquiry into equity within science funding with the House of Commons Select Committee for Science and Technology 5 .

Recently, we asked scientists on Twitter (many of whom follow the \#ScienceTwitter hashtag) to share whether they found the platform useful and why ${ }^{6}$. This received substantial attention, with $>490$ retweets, $>350$ replies, $>4300$ likes, and $>600,000$ impressions (the number of users who read and interacted with the posts). Replies covered a wide range of themes, including growing new and old collaborations, improving science practise, finding papers, sharing work and supporting colleagues. Many illustrated how their Twitter usage enabled them to feel more rounded and better connected. Some developed non-traditional skills (e.g. coding, pedagogy, listening skills), while others were able to share life and cultural experiences, and a large number spoke of improved interactions with students and colleagues and opportunities to learn best practice first-hand. The benefits of Twitter extend beyond these perks, but in essence, social media provides a voice to the scientists behind the science. People felt they could vent frustrations, share personal victories and setbacks, and seek out role models and voices who may otherwise be hidden or not heard .

Twitter is not the only option of course. Social media platforms have transformed the opportunities for marginalised communities, turning the challenges that individuals from underrepresented groups experience in academia into a chance to connect with like-minded people from around the world. In the past few years a number of truly global, special interest communities have formed, including VanguardSTEM, 500 Women Scientists, 500 Queer Scientists, \#MeTooSTEM, Pride in STEM and Minorities in STEM. These communities offer mentoring and advice to underrepresented individuals and/or early career scientists, amplify the voices and perspectives of those who are otherwise silenced, challenge dated 
institutional practices, advocate for evidence- and equity-based policies, and offer a new face for what a scientist looks like. For example, a recent investigation led by American science communicators analysed the impact of scientists' selfies on Instagram - the imagesharing site ${ }^{7}$. The findings suggest that scientists using Instagram to share selfies were perceived as warmer, more trustworthy and no less competent than their non-selfie sharing colleagues. Resources like 500 Women Scientists', Request a Woman Scientist, and Skype a Scientist are platforms that help to connect women, non-binary gender, and racial and ethnic minority experts with the public, increasing the representation of these groups to a broad range audience ${ }^{8}$.

Given the benefits we have outlined above, the question arises of how can we evaluate the impact of our social media endeavours? In a world where receiving a response from a journal or the results of a grant application can take months, the immediate feedback of social media can be validating. However, influence evaluation in the online world is difficult and quantitative metrics can be easily to overinterpreted: Professor Nic Gaston (University of Auckland) suggests that the impact of social media is more than simple metrics (e.g. clicks, likes, 'upvotes', or webpage hits), and over use of these metrics to measure value and distort how people use it as a forum for engagement ${ }^{9}$. A popular example of this is the generation of the Kardashian Index ${ }^{8}$, an humorous but inherently flawed metric (which the inventor notes lacks statistical rigour) that attempts to evaluate whether an individual's social media reach can extend beyond that allowed by your $\mathrm{H}$-index. The absurdity of such a measure is compounded if we remember that the $\mathrm{H}$-index is itself flawed ${ }^{11-14}$, and is only very loosely (if at all) linked to the 'quality' of your science. The Kardashian Index in fact reveals a significant benefit of social media: we can use social media to escape the ivory tower and reach more important and influential audiences (i.e. the general public).

In our efforts to improve the culture of academia and create a more inclusive space, we have witnessed cases where scientists on social media make use of our relative freedom to express personal opinions that oppose that of their employer. Opening these new avenues for expression and amplifying support can create tension as illustrated in 2017, when academics at the University of Leeds went on strike in response to a nebulous new employment policy (staff could be dismissal on the grounds of "Some Other Substantial Reason") ${ }^{15}$. There are concerns that such a clause might be used to clamp down on academics using social media where an employee was, for example, critical of their university ${ }^{16}$.

But beyond the potential for action initiated by our employers, being active (aka 'highly wired ${ }^{\prime 17}$ ) on social media can result in personal abuse. We are passionate and outspoken individuals, who frequently, and individually and collectively lobby for cultural change within the academia. These activities do receive universal support, and each of us has been on the receiving end of nasty attacks from the general public as well as from other academics. Fortunately, these remain relatively rare, but each case is exhausting, time-consuming and potentially dangerous, because we are individuals who can be associated with our employers, even when fighting the so-called trolls. The extent of these attacks varies, but on at least one occasion we have have sought advice from the University legal team. These moments of risk are complicated further because the ability for content to "go viral" is random, and a message can easily be perceived in a way very different to that intended. Social media increases the sharing of ideas, and the faster and cross cultural aspect of 
these interactions means that people may see more content that causes individual moral outrage $^{18}$.

So, how might we avoid these risks in the future? The 1752 group, who lobby to end staffstudent sexual harassment, have recently proposed that employees are advised not to say anything on social media that they would not feel comfortable saying at the end of a lecture ${ }^{19}$.

On balance, we value the enrichment of our personal and professional lives that social media offers, providing us with many opportunities to interact with others. For us this is sufficient motivation to continue our use of social media. In writing this article, we have found it strange, and at times outright difficult, to continue to have to justify our social media use. Other professionals gain influence, financial reward, reach, praise (and even presidencies) from social media engagement. Why should academia be stuck in the dark ages? Ultimately, if we want our institutions to excel and modernize, we had better get engaged online.

\section{Acknowledgements:}

T.B. Britton acknowledges funding of his research fellowship from the Royal Academy of Engineering. We thank Dr Emma Chapman for insightful discussions, and remind everyone that tweets often contain typographical errors associated with autocorrect. We thank our Editor, Stephen Davey, for his insight and help with this article. We also thank the 40 or so Twitter users, who provided reviewer comments, via google docs, prior to publication of this article.

\section{Endmatter:}

@bmatb

@seis_matters

@jesswade

\section{Further information:}

The Inclusion Group for Equity in Research in STEMM https://www.tigerinstemm.org/ Imperial College London Social Media Guidelines https://www.imperial.ac.uk/staff/tools-andreference/web-guide/tools/social-media/

[1] Key Q., Ahn Y-Y., Sugimoto C.R. A systematic identification and analysis of scientists on twitter Plos One (2017) https://doi.org/10.1371/journal.pone.0175368

[2] Bastian, H, "Who's Who on Science Twitter and Who Counts?" (2017) at $<$ https://blogs.plos.org/absolutely-maybe/2017/04/28/whos-who-on-science-twitter-and-whocounts/>

[3] Isabelle M. Côté, Emily S. Darling, Scientists on Twitter: Preaching to the choir or signing from the rooftops FACETS (2018) https://doi.org/10.1139/facets-2018-0002 [4] http://blogs.rsc.org/rscpublishing/2018/12/10/rsc-twitter-poster-conference-2019/ [5] House of Commons Science and Technology Committee (2019) 'My Science Inquiry' Sixteenth Report of Session 2017-2019 HC 1716. 
[6] Britton, B, "Raise your hand and share if you are a scientist and have found twitter useful. If you want to share why, that would be amazing too." (2019). at <https://twitter.com/BMatB/status/1123820553389916165>

[7] Jarreau, P., Cancellare, I., Carmichael, B., Porter, L., Toker, D., \& Yammine, S. Plos One (2019). Using Selfies to Challenge Public Stereotypes of Scientists.

https://doi.org/10.1371/journal.pone.0216625

[8] Mccullagh, E.A., Nowak, K, Pogoriler, A., Metcalf, J.L., Zaringhalam, M., Zelikova, T.J. Request a woman scientist: A database for diversifying the public face of science Plos Biology (2019) https://doi.org/10.1371/journal.pbio.3000212

[9] Gaston, Nicola (@nicgaston) "Was a privilege and a pleasure to get to speak @AuklandUni science graduation today - here are my notes fwiw \#peerreview" (2019) at $<$ https://twitter.com/nicgaston/status/1123437220990472192>

[10] Hall, N. The Kardashian index: a measure of discrepant social media profile for scientists Genome Biology (2014) https://doi.org/10.1186/s13059-014-0424-0

[11] Barnes, C. The H-index Debate: An Introduction for Librarians The Journal of Academic Librarianship (2017) https://doi.org/10.1016/j.acalib.2017.08.013

[12] Rownland, I. Is it time to bury the H-index? The Bibliomagician (2018) at $<$ https://thebibliomagician.wordpress.com/2018/03/23/is-it-time-to-bury-the-h-index/>

[13] Minasny, B., Hartemink, A.E., McBratney, A., Jang, H.-J. Citations and the h index of soil researchers and journals in the Web of Science, Scopus, and Google Scholar PeerJ (2013) https://peeri.com/articles/183/

[14] Malesios, C. C., Psarakis, S. Comparison of the h-index for different fields of research using bootstrap methodology Quality and Quantity (2014)

https://link.springer.com/article/10.1007/s11135-012-9785-1

[15] (2019) at <http://speakout.web.ucu.org.uk/university-of-leeds-statutes-no-sackerscharter/>

[16] Swansea University, Social Media Guidelines (2019) at <https://www.swansea.ac.uk/media/Social\%20Media\%20Guidelines.pdf>

[17] Grand, Ann; Holliman, Richard; Collins, Trevor and Adams, Anne (2016). "We muddle our way through": shared and distributed expertise in digital engagement with research. Journal of Science Communication, 15(4) pp. 1-23.

http://oro.open.ac.uk/46686/1/JCOM 15042016 A05.pdf

[18] Crockett M.J. Moral outrage in the digital age Nature Human Biology (2017) https://doi.org/10.1038/s41562-017-0213-3

[19] Chapman, Emma "So this might be unpopular but we've been talking about this in the @1752Group and came to the conclusion that employee's should be advised to not say anything on social media that they wouldn't feel comfortable saying in a chat at the end of a lecture."

(2019) at <https://twitter.com/DrEOChapman/status/1126575152412672001> 